\title{
Treatment of Open-Angle Glaucoma and Ocular Hypertension with Preservative-Free Tafluprost/ Timolol Fixed-Dose Combination Therapy: The VISIONARY Study
}

Francesco Oddone · Lucia Tanga · Péter Kóthy · Gábor Holló •

The VISIONARY Study Group

Received: December 23, 2019 / Published online: February 18, 2020

(c) The Author(s) 2020

\section{ABSTRACT}

Introduction: A non-interventional, multicenter, European, prospective evaluation of the effectiveness, tolerability, and safety of a topical preservative-free tafluprost $(0.0015 \%)$ and timolol $(0.5 \%)$ fixed-dose combination (PF tafluprost/timolol FC) in adults with open-angle glaucoma (OAG) and ocular hypertension (OHT) demonstrating insufficient response to topical beta-receptor blockers or prostaglandin analogue (PGA) monotherapy.

Methods: Mean intraocular pressure (IOP) change from baseline was measured at study visits following a switch to PF tafluprost/timolol FC. Primary endpoint was absolute mean IOP change at month 6 . Change from baseline concerning ocular signs and symptoms was also explored.

The members of The VISIONARY Study Group are listed in Acknowledgements.

Enhanced digital features To view enhanced digital features for this article go to https://doi.org/10.6084/ m9.figshare.11627361.

F. Oddone $(\bowtie) \cdot$ L. Tanga

IRCSS-Fondazione Bietti, Rome, Italy

e-mail: oddone-bietti@gmail.com

P. Kóthy · G. Holló

Department of Ophthalmology, Semmelweis

University, Budapest, Hungary
Results: Analyses included 577 patients (59.6\% female). Mean age (SD) was 67.8 (11.67) years. Mean (SD) IOP reduction from baseline was significant at all study visits; 5.4 (3.76) $\mathrm{mmHg}$ (23.7\%) at week 4, $5.9(3.90) \mathrm{mmHg}(25.6 \%)$ at week 12, and 5.7 (4.11) $\mathrm{mmHg}(24.9 \%)$ at month 6 ( $p<0.0001$ for all visits). At month 6 , $69.2 \%, 53.6 \%, 40.0 \%$, and $25.8 \%$ were responders based on $\geq 20 \%, \geq 25 \%, \geq 30 \%$, and $\geq 35 \%$ cutoff values for mean IOP, respectively. Significant reductions were observed concerning corneal fluorescein staining $(p<0.0001)$, dry eye symptoms, irritation, itching, and foreign body sensation ( $p<0.001$ for each parameter). Conjunctival hyperemia was significantly reduced at all study visits ( $p<0.0001$ at each visit). Overall, 69 treatment-related adverse events (AEs) were reported, one of which was serious (status asthmaticus). Most AEs were mild to moderate in severity, and the majority had resolved or were resolving at the end of the study period.

Conclusion: In clinical practice, PF tafluprost/timolol FC provided statistically and clinically significant IOP reductions in patients with OAG and OHT insufficiently controlled on or intolerant to PGA or beta-receptor blocker monotherapy. The full IOP reduction appeared at week 4 and was maintained over the 6 -month study period. Key symptoms of ocular surface health improved.

Trial Registration: European Union electronic Register of Post-Authorisation Studies (EU PAS) register number, EUPAS22204. 
Keywords: Fixed-dose combination; Ocular hypertension; Open-angle glaucoma; Ophthalmology; Preservative-free topical medication; Tafluprost; Timolol

\section{Key Summary Points}

Why carry out this study?

Prostaglandin analogue (PGA) and betareceptor blocker combination therapies are among the most extensively used intraocular pressure (IOP)-lowering treatments in glaucoma care. Fixed-dose combinations simplify the treatment regimen and reduce the number of daily instillations, compared with administration of the corresponding concomitant medications, and preservative-free formulations are generally considered to offer improved tolerability.

The VISIONARY study aimed to provide real-world data concerning the treatment effectiveness of the preservative-free tafluprost $(0.0015 \%)$ and timolol $(0.5 \%)$ fixed-dose combination ( $\mathrm{PF}$ tafluprost/timolol FC) in people with open-angle glaucoma (OAG) and ocular hypertension (OHT) who demonstrated an insufficient response to previous monotherapy treatment with topical betareceptor blocker or PGA monotherapy.

\section{What was learned from the study?}

Mean (SD) IOP reduction from baseline for patients switched to PF tafluprost/timolol FC from PGA or timolol therapy was 5.4 (3.76) $\mathrm{mmHg}(23.7 \%)$ at week 4, $5.9(3.90) \mathrm{mmHg}$ (25.6\%) at week 12 and 5.7 (4.11) $\mathrm{mmHg}$ $(24.9 \%)$ at month 6 ( $p<0.0001$ for all visits).

In routine clinical practice, $\mathrm{PF}$ tafluprost/timolol FC provided statistically and clinically significant IOP reduction already at week 4 , and the efficacy was maintained over 6 months in patients with OAG and OHT insufficiently controlled on PGAs or beta-receptor blockers.
Key symptoms of ocular surface health were improved, compared with previous PGA or beta-receptor blocker monotherapy, and treatment was generally well tolerated.

\section{INTRODUCTION}

Elevated intraocular pressure (IOP) is the most important modifiable risk factor for development and progression of open-angle glaucoma (OAG) [1]. A wealth of data has demonstrated that IOP reduction is associated with slowing of disease progression and visual field impairment; IOP reduction is thus essential for the preservation of vision in glaucoma [1-5].

Topical IOP-lowering medication remains the mainstay of glaucoma therapy [1-5]. Strong IOP-lowering efficacy, a simplified instillation regimen, and good topical and systemic tolerance are all essential for optimal treatment outcomes and long-term adherence with topical glaucoma medications $[6,7]$. When monotherapies do not reach the target IOP, combination therapy is necessary [6]. Prostaglandin analogue (PGA) and beta-receptor blocker (typically timolol $0.5 \%$ ) combination therapies are among the most extensively used medications in glaucoma care $[6,8]$. Fixed-dose combination (FC) formulations are frequently used in preference to the administration of the corresponding concomitant medications, since they simplify the treatment regimen and reduce the number of daily instillations as well as the total amount of preservatives applied to the eye $[1,6,7,9-11]$.

Preservative-free (PF) topical glaucoma therapy has become increasingly used in the management of glaucoma worldwide [7, 10-18]. Preservatives, and in particular benzalkonium chloride (BAK), the most commonly used preservative, have been shown to induce and seriously worsen ocular surface disease (OSD) [7, 19-24]. Preservative-induced OSD is a major problem in the long-term topical treatment of 
glaucoma, affecting $45-60 \%$ of chronically treated glaucomatous eyes [7, 25, 26]. PF medication is potentially advantageous for all glaucomatous eyes because the BAK-induced ocular surface toxicity worsens with the duration of preserved topical treatment [7, 19-27]. The overall quantity of BAK used throughout a patient's lifetime is also inversely related to filtration surgery success [7, 19-27]. The fixed combination of tafluprost $0.0015 \%$ and timolol $0.5 \%$ was one of the first PF PGA/timolol FCs used for the treatment of OAG and ocular hypertension (OHT) [10, 14-18]. Randomized controlled trials demonstrated a low rate of conjunctival hyperemia with PF tafluprost/timolol FC treatment [14, 15]. A recent 24-h investigation also showed that PF tafluprost/timolol FC was associated with less frequent and less severe hyperemia compared with the prestudy period on preserved latanoprost treatment [10].

Randomized, prospective, controlled investigations remain the gold standard for robust assessment of efficacy and safety data for regulatory purposes. However, as a result of their inclusion and exclusion criteria, the full spectrum of routine clinical practice cannot be explored in such investigations. Regarding topical glaucoma medications, observational studies may provide additional information on tolerance and real-life effectiveness since these investigations also report on patients with OSD or other relevant comorbidities that represent exclusion criteria in most randomized, prospective, clinical studies. In addition, observational studies reflect real-world experience concerning medication change without prior treatment washout. Therefore, real-world evidence is becoming increasingly welcomed by regulatory bodies [28-30].

In the current investigation, systematically registered clinical data were used to evaluate the IOP-lowering effectiveness and tolerability of the PF tafluprost/timolol FC over a 6-month period in participants with OAG and OHT, who were previously treated with either a topical PGA or a topical beta-receptor blocker monotherapy.

\section{METHODS}

\section{Study Design and Visit Schedule}

This was a 6-month, observational, multicenter, European, prospective clinical study. In line with European Medicines Agency (EMA) requirements, the study was registered under the European Network of Centres for Pharmacoepidemiology and Pharmacovigilance $\left(\mathrm{ENCePP}^{\circledR}\right)$ European Union electronic Register of Post-Authorisation Studies (EU PAS Register) (EU PAS register number EUPAS22204). The study complied with the principles of the Declaration of Helsinki. All patients included were required to provide written informed consent prior to their enrollment. The study protocol was approved by the institutional review board (IRB) or independent ethics committee (IEC) at each center/institution. The study centers/institutions are listed in the Acknowledgments alongside the relevant principal investigator.

Data were prospectively collected during routine visits, between 10 April 2017 and 9 January 2019, at 66 ophthalmology clinics in Austria, Denmark, Hungary, Ireland, Italy, Latvia, Netherlands, Norway, Russia, Spain, Sweden, and the UK. For patients, attendance was mandatory only for the baseline and month 6 study visits. However, data were recorded during interim study visits at week 4 and week 12 for participants who chose to attend these visits.

Baseline measures were recorded under topical PGA or beta-receptor blocker medication within 7 days prior to therapy change to PF tafluprost/timolol FC. Variables were documented for each eye separately at baseline and at study visits following initiation of $\mathrm{PF}$ tafluprost/timolol FC treatment. Where data on both eyes were available, the eye with the higher baseline IOP value was selected for analysis (study eye).

\section{Patient Population}

Male/female adult patients (aged $\geq 18$ years) with a diagnosis of OAG or OHT were included in the study. Participants had to be on a PGA or a beta-receptor blocker monotherapy at time of 
inclusion. They had to have medically recorded insufficient IOP control or poor tolerance on the beta-receptor blocker or PGA monotherapy prior to enrollment, which necessitated the use of a combination therapy, and the patients had to be considered likely to benefit from PF drops formulation, according to the judgement of the investigator ophthalmologist. It was possible for the investigator to indicate more than one reason for patient selection in the case report form. The category of reasons for indicating $\mathrm{PF}$ tafluprost/timolol FC comprised insufficient IOP control or progression of glaucoma on the current monotherapy, conversion of OHT to OAG, poor local tolerance of the current topical medication, insufficient adherence to the medication used, or "other reasons".

The inclusion criteria required that the participants had not undergone ophthalmic surgery within 6 months prior to the study period and had never received previous $\mathrm{PF}$ tafluprost/timolol FC treatment. Patients who were pregnant or breastfeeding at the screening visit and those with any contraindication against tafluprost or timolol treatment according to the approved licensed indication and the summary of product characteristics were not allowed to enter the study.

\section{Treatment}

During the 6-month study period, the participants treated their affected eye(s) with PF tafluprost/timolol FC (one drop daily, instilled either in the morning or in the evening). The instillation time (morning or evening) was recorded at all study visits.

\section{Efficacy Variables}

The primary endpoint was absolute mean IOP change from baseline at month 6 , following the initiation of PF tafluprost/timolol FC treatment, measured with Goldmann applanation tonometry in accordance with routine clinical practice [6]. Secondary endpoints comprised mean IOP change from baseline at interim visits, responder rate, change in clinical signs, and severity of subjective symptoms.
Responders were defined as patients with IOP change from baseline of $20 \%$ or more at week 12. In addition, the responder rate was also explored at week 4 and month 6 . Other cutoff points for change in mean IOP that were explored at week 4 , week 12 , and month 6 comprised 25\%, 30\%, and 35\%.

Subanalyses of IOP data comprised mean IOP change from baseline according to the diagnostic group (containing $>10$ patients), the type of previous medication (PGA or beta-receptor blocker), the PGA molecule (latanoprost, bimatoprost, travoprost, and tafluprost) for those using a PGA at baseline, the reported reasons for the PF tafluprost/timolol treatment initiation, instillation time (morning or evening), and the presence or absence of dry eye symptoms.

The clinical (ocular) signs were evaluated at month 6 and were compared with the baseline measures in all participants. Conjunctival hyperemia and best corrected visual acuity (BCVA) data reporting was mandatory at all study visits. Visual acuity data were collected in decimal, logMAR, or fraction (foot or meters) scales (according to the sites' practice) and were converted to the decimal scale using the appropriate conversion formulas [31]. Data concerning corneal fluorescein staining (CFS) were reported using the Oxford grading scale $(0-\mathrm{V}$ grade dependent on intensity of punctate staining across the cornea and conjunctiva) [32]. Schirmer's test, tear film breakup time (TBUT), and conjunctival hyperemia were collected at baseline and month 6; however, these evaluations were allowed as optional tests at interim visits. Conjunctival hyperemia was evaluated using a four-grade severity scale (none, mild, moderate, and severe).

The subjective symptoms were assessed at each study visit. These comprised dry eye feeling, irritation, itching, foreign body sensation, and eye pain. Symptom severity was evaluated using a four-grade scale (none, mild, moderate, and severe). In addition, the investigator provided evaluation concerning the effectiveness of the PF tafluprost/timolol FC therapy, the clinical signs, and compliance to the study medication compared with those for the prior monotherapy using a three-grade scale: better 
than prior medication, same as prior medication, worse than prior medication. Patients reported their assessment of tolerability concerning PF tafluprost/timolol FC treatment on a four-grade scale: very good, good, satisfactory, poor.

Reported adverse events (AEs) and treatment-related AEs were collected and documented at all visits and for the total study period.

\section{Statistical Analysis}

ICON Plc (Dublin, Ireland) conducted all statistical analyses on behalf of the VISIONARY study group. Data distribution was assessed using $Q-Q$ plots, histograms, and the Shapiro-Wilk or the Kolmogorov-Smirnov test, as needed. For normally distributed data, the mean and standard deviation (SD) are presented and the paired $t$ test was used for the comparisons. For data not normally distributed, the median values and the interquartile range (IQR) are shown and the Wilcoxon signed rank test was used to assess change in median from baseline. A linear mixed model was run with IOP as the dependent variable and all time points as independent variables to investigate time-dependent IOP changes. Time-independent comparisons between IOP values at each study visit and the baseline value utilized a paired $t$ test, taking account of repeated measures. Change from baseline concerning CFS, conjunctival hyperemia, and subjective symptoms was assessed using the Bhapkar test. The Bhapkar test can be used in marginal homogeneity and it assumes that the changes are non-directional [33].

\section{RESULTS}

\section{Study Population Demographics}

In total, 721 participants were screened for inclusion in the study and 713 were treated with the PF tafluprost/timolol FC. Of these, 577 went on to complete the 6-month visit and were included in the analysis (Fig. 1).
The baseline study population demographics are shown in Table 1. Mean age (SD) was 67.8 (11.67) years (range 23.7-96.1 years), and 59.6\% of the final population were female. The most common diagnostic groups comprised primary open-angle glaucoma (POAG; 73.7\%), OHT (19.1\%), pseudoexfoliative glaucoma (3.3\%), and normal tension glaucoma (2.3\%). The majority of the patients $(72.1 \%)$ changed medication from a PGA and $27.9 \%$ from beta-receptor blocker (timolol) therapy. At baseline, the mean (SD) IOP was 21.55 (4.45) $\mathrm{mmHg}$, the mean (SD) CFS score was 0.76 (0.94), and median BCVA decimal score (IQR) was $0.9(0.4)$.

\section{IOP Change from Baseline}

Concerning the primary endpoint, the absolute mean IOP change at month 6 was statistically significant $(p<0.0001)$ (Table 2$)$. Mean (SD) IOP at month 6 was $15.8 \mathrm{mmHg}$ (3.21). Mean (SD) IOP reduction from baseline was 5.7 (4.11) $\mathrm{mmHg}$, representing an overall IOP reduction of $24.9 \%$. Statistically significant mean IOP reductions from baseline were observed at each of the interim study visits. At week 4 , it was 5.4 (3.76) $\mathrm{mmHg}(23.7 \% ; p<0.0001)$, and 5.9 (3.90) $\mathrm{mmHg}(25.6 \% ; p<0.0001)$ at week 12 . At month $6,69.2 \%, 53.6 \%, 40.0 \%$, and $25.8 \%$ of participants were responders based on $\geq 20 \%$, $\geq 25 \%, \geq 30 \%$, and $\geq 35 \%$ cutoff values for change in mean IOP, respectively (Fig. 2).

\section{Intraocular Pressure Subanalyses}

The mean (SD) IOP at baseline was 21.5 (5.00) $\mathrm{mmHg}, 22.3$ (4.30) $\mathrm{mmHg}, 17.2$ (2.68) $\mathrm{mmHg}$, and $21.7(4.13) \mathrm{mmHg}$ for patients diagnosed with POAG, OHT, normal tension glaucoma, and pseudoexfoliative glaucoma, respectively. At month 6, respective relative IOP reductions were $25.4 \%, 26.1 \%, 15.8 \%$, and $17.6 \%$. Differences between subgroups did not reach statistical significance $(p \geq 0.324)$.

At baseline, mean (SD) IOP was 21.9 (4.36) $\mathrm{mmHg}$ and 21.4 (4.48) $\mathrm{mmHg}$ for the beta-receptor blocker and PGA users, respectively. At month 6 IOP was 15.3 (3.10) $\mathrm{mmHg}$ in those previously using a beta-receptor blocker, representing 6.6 (4.16) $\mathrm{mmHg}(28.5 \%)$ reduction 


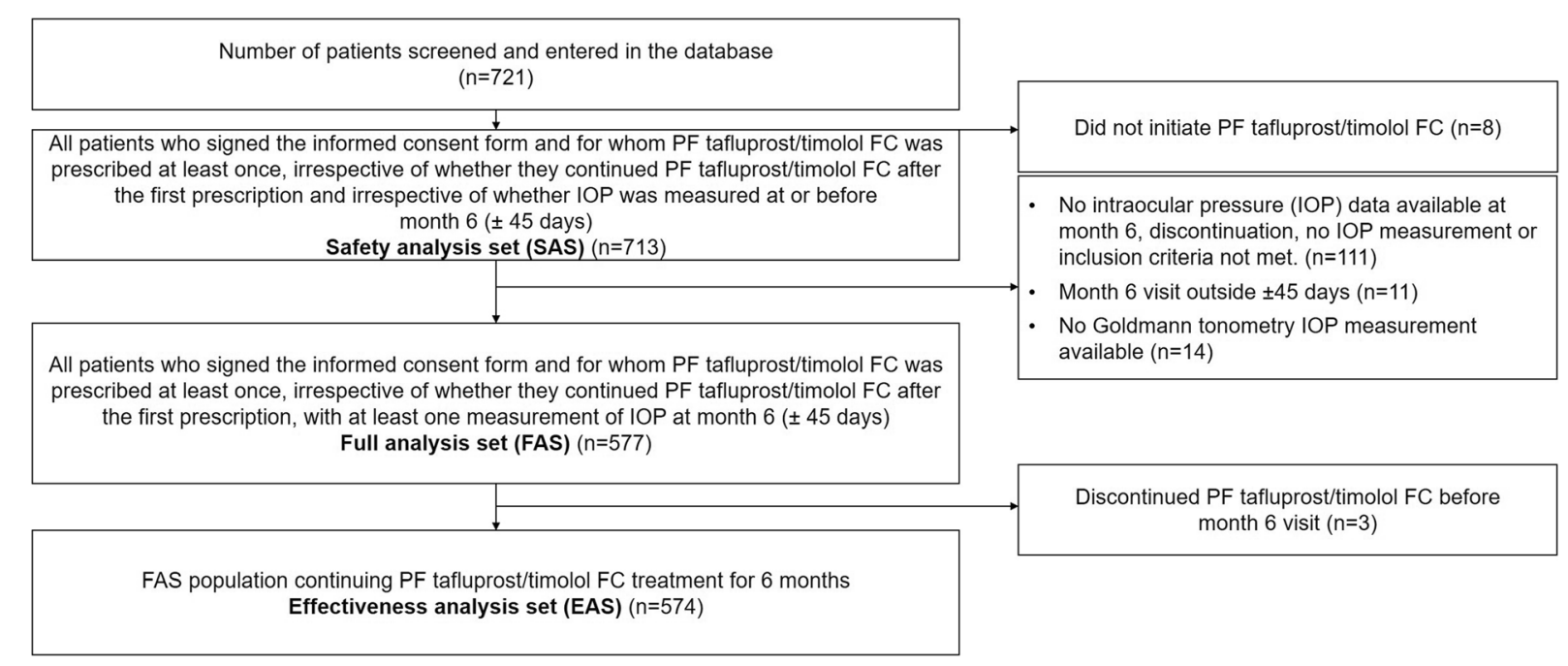

Fig. 1 Patient disposition

from baseline $(p<0.0001)$. In those treated with a PGA, at month 6 IOP was $16.0(3.23)$ $\mathrm{mmHg}$, representing 5.4 (4.04) $\mathrm{mmHg}(23.6 \%)$ reduction $(p<0.0001)$.

Prior to the study period, $64,63,87$, and 201 patients were on bimatoprost, travoprost, tafluprost, and latanoprost, respectively (for one patient in the study population, the specific PGA used was not recorded). The IOP at baseline was 20.6 (4.39) $\mathrm{mmHg}, 20.0$ (3.90) $\mathrm{mmHg}, 20.8$ (3.29) $\mathrm{mmHg}$, and 22.3 (4.93) $\mathrm{mmHg}$ for participants treated with bimatoprost, travoprost, tafluprost, and latanoprost, respectively. At month 6 , respective mean relative IOP reductions were $20.5 \%, 21.3 \%, 22.7 \%$, and $25.9 \%$ ( $p<0.0001$ for all groups).

Of the 577 participants included in the analysis, 469 individuals (81.3\%) changed to PF tafluprost/timolol FC treatment because of uncontrolled IOP, and 106 (18.4\%) were enrolled because of poor ocular tolerance on the previous topical IOP-lowering medication (irrespective of the success of the IOP control). At month 6 , the mean relative IOP reduction was $26.6 \%$ for participants with insufficient IOP control at baseline and $19.6 \%$ for those who did not tolerate the previous topical medication well $(p<0.001)$.

At month 6, the instillation time (morning versus evening) was recorded for 521 patients. The mean (SD) relative IOP reduction from baseline was 5.50 (4.14) $\mathrm{mmHg}$ (23.6\%) for those using morning instillation and 5.67 (3.99) $\mathrm{mmHg}(24.7 \%)$ for evening instillation $(p=0.5966)$. The mean relative IOP reduction was not significantly different when comparing those who reported no dry eye symptom at baseline $(n=242)$ and those $(n=224)$ who reported any severity of dry eye symptom (24.9\% vs $24.4 \%$ at month $6, p=0.754)$.

\section{Clinical Signs}

Compared with the baseline value, the mean (SD) CFS score was reduced at all study visits during the PF tafluprost/timolol FC treatment (Table 3). At baseline, the median (IQR) Schirmer's test result was 10.0 (8.0). No statistically significant change was seen concerning the Schirmer's test result during the study period $(p=0.258)$. TBUT was $6.0(5.0) \mathrm{s}$ at baseline and increased by an average $1 \mathrm{~s}$ value at all later study visits $(p=0.0035)$. Statistically significant increases in median TBUT were observed at each study visit for participants on baseline PGA monotherapy ( $p=0.0012)$ but not in beta-receptor blocker users $(p=0.7490)$.

CFS scores of 0 or 1 were found in 188 (80.3\%) patients at baseline and in 249 (91.5\%) at month 6 among those with data available. The mean (SD) CFS score at month 6 was 0.47 (0.71), which was a statistically significant 
Table 1 Demographics of the participants

\begin{tabular}{|c|c|}
\hline \multicolumn{2}{|l|}{ Sex, $n(\%)$} \\
\hline Male & $233(40.4)$ \\
\hline Female & $344(59.6)$ \\
\hline \multicolumn{2}{|l|}{ Age (years) } \\
\hline Mean \pm SD & $67.8 \pm 11.67$ \\
\hline Range & $23.7-96.1$ \\
\hline \multicolumn{2}{|l|}{ Diagnosis, $n(\%)$} \\
\hline POAG & $425(73.7)$ \\
\hline $\mathrm{OHT}$ & $110(19.1)$ \\
\hline Pseudoexfoliative glaucoma & $19(3.3)$ \\
\hline Normal tension glaucoma & $13(2.3)$ \\
\hline Pigmentary glaucoma & $5(0.9)$ \\
\hline Other glaucoma & $5(0.9)$ \\
\hline \multicolumn{2}{|l|}{ Study eye, $n(\%)$} \\
\hline Right & $363(62.9)$ \\
\hline Left & $214(37.1)$ \\
\hline \multicolumn{2}{|l|}{ Previous treatment, $n(\%)$} \\
\hline Beta-blocker therapy & $161(27.9)$ \\
\hline PGA therapy & $416(72.1)$ \\
\hline IOP at baseline, $\mathrm{mmHg}($ mean $\pm S D)$ & $21.55 \pm 4.45$ \\
\hline $\begin{array}{l}\text { CFS score (Oxford grade scale) } \\
\quad(\text { mean } \pm S D)(n=238)\end{array}$ & $0.76 \pm 0.94$ \\
\hline $\begin{array}{l}\text { BCVA decimal score, median (IQR) } \\
\quad(n=461)\end{array}$ & $0.9(0.40)$ \\
\hline Schirmer's test, median (IQR) $(n=124)$ & $10.0(8.00)$ \\
\hline TBUT seconds, median (IQR) $(n=176)$ & $6.0(5.00)$ \\
\hline
\end{tabular}

$S D$ standard deviation, $P O A G$ primary open-angle glaucoma, $O H T$ ocular hypertension, $P G A$ prostaglandin analogue, IOP intraocular pressure, CFS corneal fluorescein staining, $B C V A$ best corrected visual acuity, TBUT tear breakup time, $I Q R$ interquartile range

reduction compared with the mean baseline value $(0.76[0.94] ; p<0.0001)$ (Table 3$)$. Overall, $32.7 \%$ of patients demonstrated a reduction in the CFS score from baseline at month 6 ,
$53.6 \%$ showed no change, and CFS was increased in $13.6 \%$ of the patients.

Statistically significant reductions, compared with baseline values, were found for conjunctival hyperemia at week 4, week 12 and month 6 $(p<0.0001$ for each study visit). At month 6 , $38.7 \%$ of the patients demonstrated reduction in conjunctival hyperemia, $50.7 \%$ showed no change, and $10.7 \%$ showed increased hyperemia. At baseline, conjunctival hyperemia was mild or absent in $79.6 \%$ of participants on latanoprost, $88.9 \%$ of those treated with tafluprost, $60.4 \%$ on bimatoprost, and $66.7 \%$ on travoprost (Fig. 3). Moderate or severe hyperemia was reported for the remaining patients at baseline. During the study period, conjunctival hyperemia was significantly reduced at all study visits for participants treated with each of the baseline PGA monotherapies; latanoprost $(p<0.0001)$, tafluprost $(p=0.029)$, bimatoprost $(p<0.0001)$, and travoprost $(p<0.0001)$. The greatest improvement in conjunctival hyperemia severity was found in participants previously treated with bimatoprost or travoprost (>59.6\% improvement at each study visit, for both medications).

\section{Subjective Symptoms}

At baseline, subjective symptoms were generally reported absent or of mild severity. This was the case for more than $80 \%$ of patients concerning dry eye, irritation, and itching, and more than $90 \%$ for individuals reporting of foreign body sensation and eye pain. However, at month 6, statistically significant reductions from baseline were reported concerning severity of dry eye, irritation, itching, and foreign body sensation $(p<0.001$ for each category). Overall, dry eye severity was reduced for $30.8 \%$ of the patients, while $59.3 \%$ of the participants reported no change, and $9.8 \%$ of the patients reported increased severity. Severity of irritation was reduced in $31.0 \%$ of the participants, while $61.0 \%$ experienced no change. Itching severity was reduced in $26.6 \%$ of participants, while $62.8 \%$ reported no change. Foreign body sensation was reduced in $23.0 \%$ of the participants, $69.9 \%$ reported no change, and an increase in 
Table 2 Intraocular pressure change from baseline at week 4, week 12, and month 6

\begin{tabular}{llllll}
\hline Visit & $\boldsymbol{N}$ & $\begin{array}{l}\text { Mean }(\mathbf{S D}) \\
\text { IOP }(\mathbf{m m H g})\end{array}$ & $\begin{array}{l}\text { Mean }(\mathbf{S D}) \text { reduction in } \\
\text { IOP from baseline }(\mathbf{m m H g})\end{array}$ & $\begin{array}{l}\text { Mean percentage reduction } \\
\text { in IOP from baseline }\end{array}$ & $\boldsymbol{p}_{\text {value }}^{*}$ \\
\hline Baseline & 577 & $21.5(4.45)$ & & & \\
Week 4 & 541 & $16.2(3.28)$ & $5.4(3.76)$ & 23.7 & $<0.0001$ \\
Week 12 & 503 & $15.7(3.98)$ & $5.9(3.90)$ & 25.6 & $<0.0001$ \\
Month 6 & 577 & $15.8(3.21)$ & $5.7(4.11)$ & 24.9 & $<0.0001$ \\
\hline
\end{tabular}

IOP intraocular pressure

\# Significance testing using two-sided paired $t$ test for change in mean IOP from baseline to week 4, week 12, and month 6

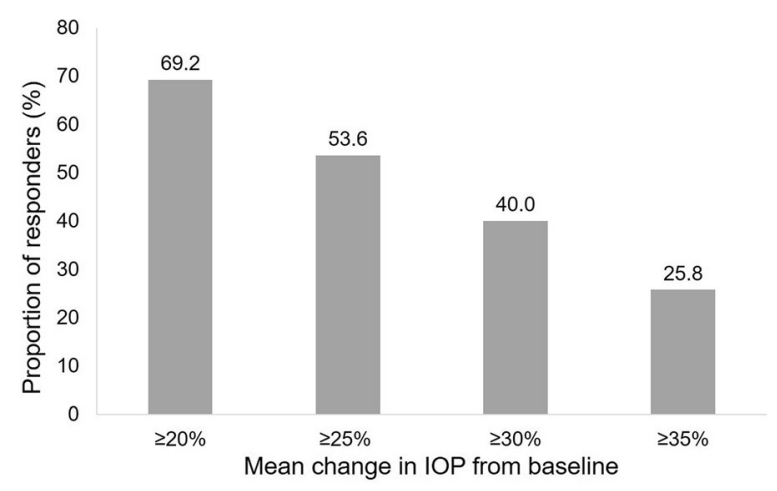

Fig. 2 Percentage of responders according to different intraocular pressure reduction cutoff values at month 6 . IOP intraocular pressure

severity was reported by $7.5 \%$ of the patients. No significant change was found concerning eye pain severity at month $6(p=0.058) ; 7.8 \%$ of the participants reported pain reduction, $88.9 \%$ experienced no change, and $3.3 \%$ reported that the pain increased.

\section{Physician Assessments}

On the basis of clinical evaluation, most investigators $(84.7 \%)$ considered IOP control more effective with PF tafluprost/timolol FC treatment than with the previous medication, at month 6. The investigators also reported reduced ocular signs at month 6 in $63.6 \%$ of the participants, compared with the previous medication. Overall, compliance to the PF tafluprost/timolol FC treatment was perceived to be greater $(48.9 \%)$ or comparable $(46.0 \%)$ with that on the previous medications, when evaluated by the investigators.

\section{Patient Assessment}

The majority of patients reported that the tolerability of the PF tafluprost/timolol FC treatment was good or very good at week $4(87.9 \%)$, week $12(92.4 \%)$, and month 6 (91.4\%).

Table 3 Cornea fluorescein staining score and its change during the study period

\begin{tabular}{|c|c|c|c|c|c|}
\hline & \multicolumn{5}{|c|}{ Mean change from baseline } \\
\hline & $\bar{N}$ & Mean (SD) & $N$ & Mean (SD) & $p$ value ${ }^{*}$ \\
\hline Baseline & 234 & $0.76(0.94)$ & & & \\
\hline Week 4 ( \pm 7 days) & 243 & $0.55(0.76)$ & 202 & $0.19(0.75)^{1}$ & 0.0003 \\
\hline Week 12 ( \pm 7 days $)$ & 226 & $0.54(0.78)$ & 189 & $0.21(0.93)^{2}$ & 0.0020 \\
\hline Month 6 ( \pm 45 days $)$ & 272 & $0.47(0.71)$ & 220 & $0.27(0.95)^{3}$ & $<0.0001$ \\
\hline
\end{tabular}

Change in median CFS at baseline and respective time point along with Wilcoxon signed rank test $p$ value were ${ }^{1} p=0.0003 ;{ }^{2} p=0.0023 ;{ }^{3} p<0.0001$

CFS corneal fluorescein staining 
(a)

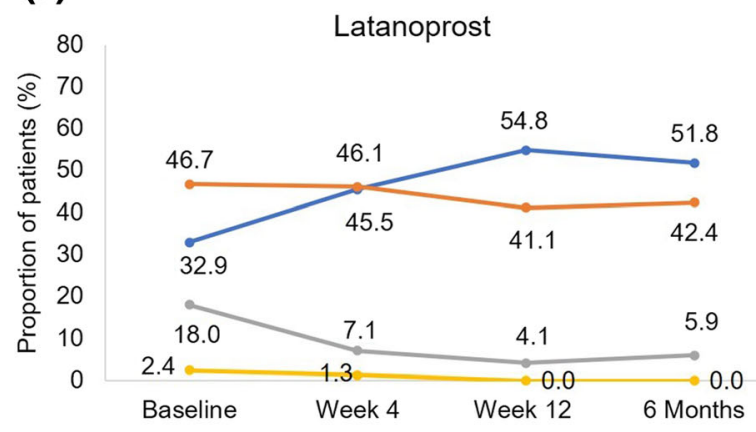

(c)

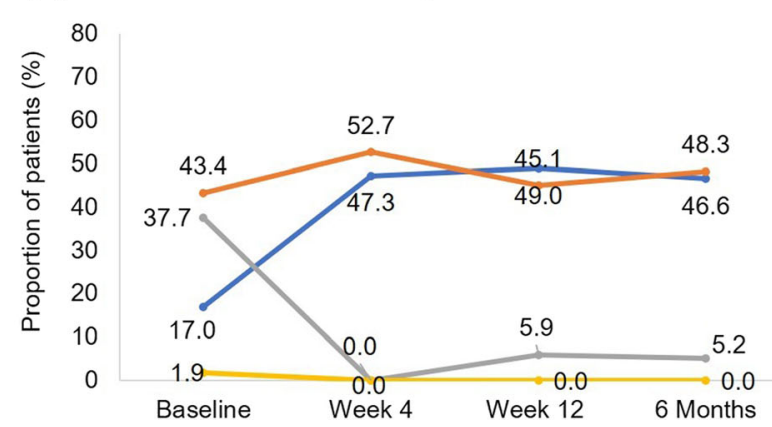

Fig. 3 Change in conjunctival hyperemia severity according to the previous prostaglandin analogue treatment. Severity of conjunctival hyperemia at each study visit for

\section{Reasons for Discontinuation}

Overall, 98 patients (17.0\%) discontinued the PF tafluprost/timolol FC treatment during the 6-month study period. The reported reasons of treatment discontinuation comprised insufficient IOP control for 2 patients, poor local tolerance for 12 patients, poor compliance for 4 patients, and "other reasons" for 13 patients. Data were missing for the remaining 67 patients.

\section{Safety}

For the 577 individuals included in the safety analysis, 129 AEs were reported by 99 (17.2\%) patients during the 6-month study period. The majority of the AEs (94.6\%) were reported as non-serious, and 69 (53.5\%) were considered to be treatment-related (Table 4). One of these was serious (status asthmaticus). Most AEs (93.0\%)
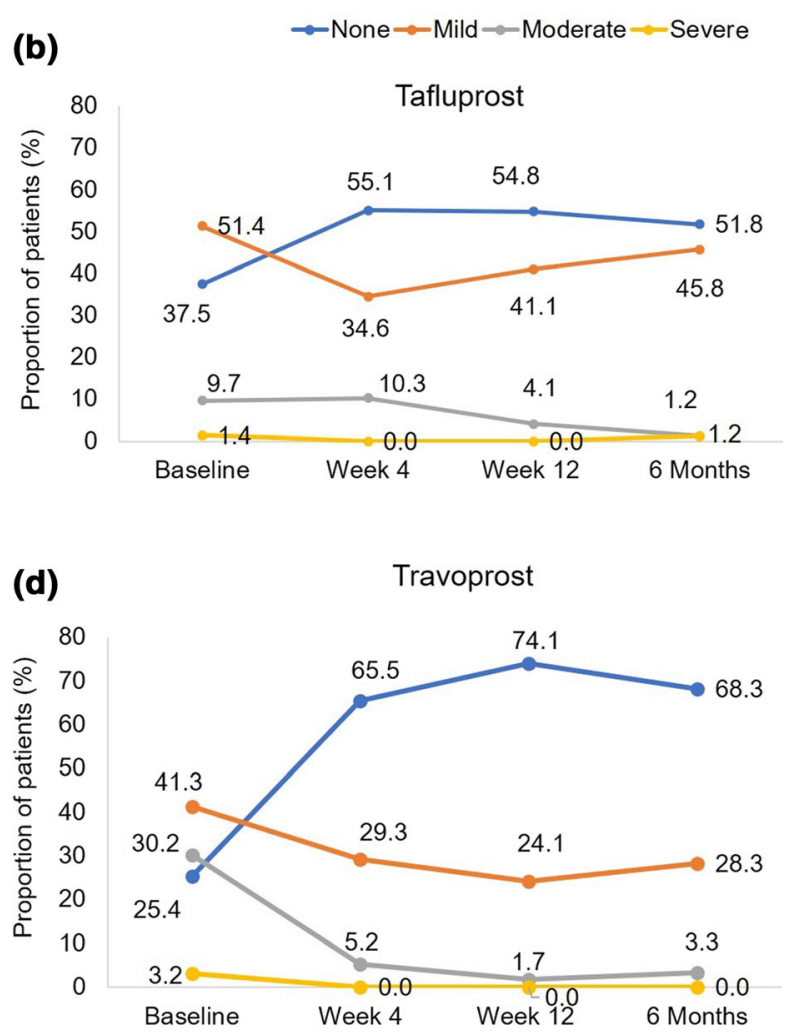

those previously treated with a latanoprost, $\mathbf{b}$ tafluprost, c bimatoprost, or $\mathbf{d}$ travoprost

were mild to moderate in severity, and the majority (71.3\%) had resolved or were resolving at the point of data cutoff.

\section{DISCUSSION}

In the current 6-month, observational, multicenter, European, prospective clinical investigation completed by 577 patients with OAG and OHT, clinical experience with changing topical medication from a PGA or a beta-receptor blocker to the PF tafluprost/timolol FC was analyzed for IOP change, objective clinical ocular surface signs, the participants' subjective symptoms, and patient- and ophthalmologistindicated satisfaction with the study medication. In this real-world study, no washout period between the prior and study medication was used, even when the prior medication was not optimally tolerated and patients with ocular 
Table 4 Treatment related adverse events reported during the study period

\begin{tabular}{ll}
\hline System/organ class & $\begin{array}{l}\text { Number of } \\
\text { treatment-related AEs }\end{array}$ \\
\hline Ocular disorders & \\
Blepharal pigmentation & 2 \\
Blepharitis & 1 \\
Conjunctival hyperemia & 1 \\
Ocular hyperemia & 4 \\
Conjunctivitis & 2 \\
Eye pain & 23 \\
Eyelid erythema & 1 \\
Eyelid edema & 1 \\
Eye Pruritis & 2 \\
Dry eye & 1 \\
Keratitis & 2 \\
Foreign body sensation & 2 \\
Eyelash growth & 1 \\
Blurred vision & 1 \\
Eye discharge & 1 \\
Ocular irritation & 1 \\
Iris hyperpigmentation & 1 \\
Lacrimation increased & 1 \\
Ocular discomfort & 2 \\
Respiratory & 1 \\
Asthma & 1 \\
Status asthmaticus & \\
Neurological & 1 \\
Headache & \\
Dizziness & \\
Cardiovascular & 1 \\
Palpitations & \\
Btrioventricular block & 1 \\
\hline
\end{tabular}

Table 4 continued

\begin{tabular}{ll}
\hline System/organ class & $\begin{array}{l}\text { Number of } \\
\text { treatment-related AEs }\end{array}$ \\
\hline Dermatological & \\
Skin hyperpigmentation & 1 \\
Abnormal hair growth & 1 \\
Rash & 1 \\
Immune disorders & \\
Hypersensitivity/allergy & 4 \\
General disorders & \\
Fatigue & 2 \\
Chest pain & 1 \\
Total & 69 \\
\hline
\end{tabular}

surface abnormalities were included. Thus, in contrast to the randomized clinical trials previously conducted on the PF tafluprost/timolol FC, in which a complete washout period was used and the patients had to be free from ocular surface abnormalities, in the current investigation we mirrored real-life clinical practice, which is particularly important to all ophthalmologists [10, 14-16].

Regarding IOP, we found a statistically and clinically significant, consistent IOP reduction at all time points including the week 4 and 12 visits, and at month 6 , which was the primary endpoint of the investigation. At month 6, for the total population, the mean IOP reduction compared to the under-treatment baseline value was $5.7 \mathrm{mmHg}(24.9 \%)$. Considering that the mean baseline IOP was $21.55 \mathrm{mmHg}$, this almost $25 \%$ IOP reduction is favorable since IOP reduction achieved with any PGA/timolol FC strongly depends on the baseline IOP, which was relatively low in our population [34]. The mean IOP reductions found in the week 4 and week 12 visits were similar, which suggests that ophthalmologists can expect a meaningful IOP reduction shortly after the change to the tafluprost/timolol FC from a PGA or beta-receptor blocker monotherapy. Furthermore, the 
responder rates were also favorable. At month 6 , almost $70 \%$ of the patients had an IOP reduction over $20 \%$, and $53.6 \%$ and $40.0 \%$ of the patients experienced an IOP reduction over $25 \%$ and $30 \%$, respectively. In almost $26 \%$ of the participants the relative IOP reduction exceeded $35 \%$. These results suggest that there is high probability of reaching a clinically satisfactory IOP reduction with changing PGA or beta-receptor blocker medication for the PF tafluprost/timolol FC in OAG and OHT.

It is also of clinical importance that meaningful IOP reduction was achieved both in OHT and all types of glaucoma represented in the investigation (POAG, pseudoexfoliative glaucoma, and normal tension glaucoma). For all these groups the relative IOP reduction ranged between $17.6 \%$ (normal tension glaucoma) and $25.4 \%$ (POAG) with no statistically significant difference between the IOP reductions in the various disease categories. Another clinically important point is that the IOP reduction in previous beta-receptor blocker users (28.5\%) was somewhat greater than that in previous PGA users (23.6\%), even if their baseline undertreatment IOP was similar $(21.4$ and $21.9 \mathrm{mmHg}$, respectively). A similar IOP reduction was found for all PGAs used before the study (20.5-25.9\%). This suggests that patients on all currently available PGA monotherapies benefit from changing to tafluprost/timolol FC, in terms of IOP reduction. Not unexpectedly, we found that those patients who were enrolled in the investigation because of insufficient IOP control on the previous monotherapy showed significantly greater IOP reduction than those participants who entered the study for poor tolerance of the previous medication (26.6\% vs. $19.6 \%)$. At the same time, however, this finding also shows that even those patients who are considered as being controlled for IOP with a PGA or a beta-receptor blocker monotherapy can gain an almost 20\% further IOP reduction by changing to the PF tafluprost/timolol FC. The similarity of IOP reduction of participants with or without dry eye symptoms at baseline (24.9\% and $24.4 \%$ at month 6) also supports that the PF tafluprost/timolol FC cannot be considered as a glaucoma medication restricted only for dry eye or OSD patients. Finally, another important result of our study is that there was no difference in mean IOP reduction between those who instilled the tafluprost/timolol FC in the morning and those who instilled it in the evening ( 5.50 vs. $5.67 \mathrm{mmHg})$. This finding is of clinical importance since it shows that, in contrast to the controlled clinical investigations in which evening instillation of tafluprost/timolol FC provided somewhat lower diurnal IOP, in real-life conditions ophthalmologists can accept their patients' preference on the instillation time [10]. This may support adherence and does not considerably influence the achieved IOP reduction.

Regarding ocular signs and symptoms, the change to PF tafluprost/timolol FC resulted in improvement in most of the measures used in our study. At month 6, the mean CFS score decreased significantly for the total study population. Almost one-third of the patients experienced a reduced CFS score. For the previous PGA users, the CFS score decreased significantly in all visits, compared to the baseline value. For the total population the mean conjunctival hyperemia score decreased significantly in all visits compared to the baseline score, and an improvement was seen in $38.7 \%$ of the patients in the month 6 visit. In the previous PGA users a highly significant reduction of hyperemia score was found for each of the four PGAs. The largest improvements were seen for the previous bimatoprost and travoprost users.

In all but one of the subjective symptom categories examined (dry eye feeling, irritation, itching, and foreign body sensation) a statistically and clinically significant improvement was reported at month 6; an improvement was found in $31.0 \%$ of the patients for dry eye feeling, $26.6 \%$ for eye itching, and $23.0 \%$ for foreign body sensation. No change in the eye pain score was found. These results show that many patients with glaucoma experienced considerable improvement of ocular tolerance on the PF tafluprost/timolol FC, even if their ocular surface-related complaints were mild or not obvious at baseline.

The evaluation of the study medication was positive by both the treating ophthalmologists and the study participants. At month 6, ophthalmologists considered IOP as better 
controlled with the PF tafluprost/timolol FC medication than with the previous monotherapy in $84.7 \%$ and classified the ocular signs as reduced compared to the baseline in $63.6 \%$. They also considered compliance to the study medication better than to the previous monotherapy in almost half of the patients, while they indicated that the compliance was similar to that on the pre-study eye drop for $46.0 \%$ of the participants. The patient-reported tolerability was similarly favorable: the frequency of good or very good tolerability category ranged between $87.9 \%$ and $92.4 \%$ in all study visits.

Early termination was recorded in 98 patients (17.0\%). As a result of the observational nature of the investigation the reason for study discontinuation was not clarified for 67 patients. The known reasons for study discontinuation were related to insufficient IOP control (2 patients), poor ocular tolerance (12 patients), and poor compliance (4 patients). Thirteen patients withdrew from the study for "other reasons". Of the 129 AEs reported by 99 patients, 69 were considered as treatment-related. Ninety-three percent of the AEs were mild and $73.1 \%$ had recovered or were recovering when the study was completed. Only one serious AE (status asthmaticus) was recorded, which probably could have been prevented by considering the contraindications of timolol, the beta-receptor blocker part of the study medication. The above safety data show that in general PF tafluprost/timolol FC was well tolerated.

Our study has limitations. It was an observational study; therefore, relatively many participants left the study without letting the investigators know about the reason for their early termination. The participants represented various areas of Europe, but their ethnicity was not investigated. Therefore, caution is needed when conclusions from our data are applied to patients with non-European origin. Since we investigated adult patients with OAG and OHT our results cannot be applied to angle-closure glaucoma and childhood glaucomas. We did not investigate switching to PF tafluprost/timolol FC from combined topical medication; therefore, our IOP results may not be reproduced when patients are switched from a fixed or unfixed topical combined medication regimen to PF tafluprost/timolol FC.

\section{CONCLUSION}

In our large multicenter observational study PF tafluprost/timolol FC demonstrated statistically and clinically significant IOP reductions in patients with OAG and OHT who were either insufficiently controlled on a PGA or beta-receptor blocker monotherapy, or did not tolerate these medications. The full IOP reduction was present already at the week 4 visit, and the efficacy was maintained over the total 6-month study period. The severity of most clinical signs and subjective symptoms decreased significantly compared to that on the previous treatment, and in general the PF tafluprost/timolol FC was well tolerated.

\section{ACKNOWLEDGEMENTS}

The authors thank the participants for their valuable contribution to the VISIONARY study. Claudia Fassari, Feride Sahin and Gabriela Saborio provided input concerning the design and implementation of the study as well as the analyses and reporting of data on behalf of Santen.

Funding. Funding was provided by Santen SA for the study, medical writing services and Rapid Service Fees. All authors had full access to all of the data in this study and take complete responsibility for the integrity of the data and accuracy of the data analysis. The contribution of IRCCS Fondazione Bietti to this work was supported by the Italian Ministry of Health and by Fondazione Roma.

Medical Writing. Medical writing services were provided on behalf of the authors by Rebecca Down at Copperfox Communications Limited, which was funded by Santen SA.

Authorship. All named authors meet the International Committee of Medical Journal Editors (ICMJE) criteria for authorship for this 
article, take responsibility for the integrity of the work as a whole, and have given their approval for this version to be published.

VISIONARY Study Group Principal Investigators. Austria: Christoph Faschinger (LKH Universitaetsklinikum Graz); Sweden: Enping Chen (St Eriks Eye Clinic, Stockholm); Hungary: Gabor Holló (Semmelweis University, Budapest), Gabor Nemeth (Borsod-Abaúj-Zemplén Megyei Kórház Szemészeti, Miskolc), Gyorgy Bator (Markusovszky Egyetemi Oktató Kórház, Szombathely), Alexis Tsorbatzoglou (Szabolcs-Szatmár-Bereg County Hospital and University Teaching Hospital, Nyíregyhaza),Tamas Acs (Bács-Kiskun Megyei Kórház, Kecskemét), Maria Ferencz (Szent Imre Egyetemi Oktatókórház, Budapest), Zoltán Sohajda (Kenézy Gyula Kórház és Rendelöintéze, Debrecen), Jeno Toth (Fejér Megyei Szent György Egyetemi Oktató Kórház, Székesfehérvár), Veronika Volner (Uzsoki Utcai Kórház, Budapest), Gabor Vogt (Magyar Honvédség Egészségügyi Központ, Budapest), Zsolt Biro (PTE- Szemészeti Klinika, Pécs), Andrea Facskó (Szegedi Tudományegyetem Szent-Györgyi Albert Klinikai Központ, Szent-György), János Nemes (Megyei Flór Ferenc Kórház, Kistarcsa), Andras Berta (University of Debrecen, Debrecen), Ilona Elek (Bugát Pál Kórház, Gyöngyös); Ireland: Eugene Ng (The Whitfield Clinic Butlerstown, Waterford); Italy: Francesco Oddone (IRCSS-Fondazione Bietti, Rome), Gemma Rossi (IRCCS-Fondazione Policlinico San Matteo, Pavia), Luca Rossetti (Ospedaliera San Paolo, Milan), Michele Vetrugno (Università di Bari, Bari), Michele Iester (Università Degli Studi Di Genova, Genoa), Giorgio Marchini (Ospedale Civile Maggiore Borgo Trento, Verona), Vincenzo Scorcia (Università degli studi Magna Græcia, Catanzaro), Giovanni Staurenghi (Ospedale Luigi Sacco, Milan), Carlo Cagini (Università degli Studi di Perugia, Perugia), Tommaso Salgarello (Fondazione Policlinico Gemelli, Rome), Paolo Bettin (IRCCS Ospedale San Raffaele, Milan), Michele Figus (Ospedale Cisanello, Pisa), Gian Luca Scuderi (Ospedale Sant'Andrea, Rome), Stefano De Cilla (Azienda Ospedaliera Universitaria Maggiore della Carita Presidio Ospedaliero San Rocco, Novara);
Latvia: Iveta Grundmane (Grund-opt Ltd, Valmiera), Nora Linavska (LENS Ltd, Liepaja), Lasma Volksone (Lavolks Ltd, Riga), Guna Laganovska (P. Stradiņš Clinical University Hospital, Riga), Kristine Baumane (Riga East Clinical University Hospital, Riga); Netherlands: Hans Lemij (Rotterdam Ophthalmic Institute, Rotterdam); Norway: Kjell Gunnar Gundersen (Dr Kjell Gunnar Gundersen MD, Haugesund); Russia: Marina Zimina (LLC Vzglyad, Leningradskaya), Valery Erichev (Federal State Budgetary Scientific Institution Scientific, Research Institute of Eye Diseases, Moscow), Elmira Adbulaeva (State Autonomous Institution of Healthcare Republican Clinical Ophthalmological Hospital of the Ministry of Healthcare of the Republic of Tatarstan, Republic of Tatarstan), Elena Karlova (State Budgetary Institution of Healthcare Samara Regional Clinical Ophthalmological Hospital, Samara), Ekaterina Zakharova (RKOB Sverdlova, Yakutsk), Irina Panova (S. Fyodorov Eye Microsurgery Federal State Institution, Saint Petersburg), Boris Malyugin (Sv. Fyodorov's Eye Microsurgery Complex, Moscow); Spain: Inaki Rodriguez Aguirrec (H.U. Donostia, Guipuzcoa), Fernando Lopez-Lopez (Instituto Oftalmologico Gomez-Ulla, Galicia), Antonio Moreno Valladares (Hospital Ntra. Sra. del Perpetuo Socorro, Albacete), Javier Benitez del Castillo (Hospital Universitario de Jerez, Cadiz), Rafael Gimenez (Hospital Reina Sofia, Cordoba), Maria Parrilla Vallejo (Hospital La Macarena, Seville), Jose Garcia Medina (Hospital General Universitario Morales Meseguer, Murcia), Alfonso Anton Lopez (Institut Català de Retina, Barcelona), Sergio Torregrosa (Hospital Punta de Europa, Cadiz), Jorge Loscos (Hospital German Trias i Pujol, Barcelona); Denmark: Miriam Kolko (Rigshospitalet Valdemar Hansensvej, Glostrup); UK: Ejaz Ansari (Maidstone Hospital Hermitage, Maidstone, Kent), David Broadway (Norfolk and Norwich University Hospital, Norwich, Norfolk), Katharine Claridge (Royal Cornwall Hospital, Truro, Cornwall), Simon Ruben (Southend University Hospital NHS Foundation Trust, Westcliff-On-Sea, Essex), James Kirwan (Queen Alexandra Hospital, Portsmouth Hospitals NHS Trust, Portsmouth, Hampshire), Anca Nita (Royal Victoria 
Infirmary, The Newcastle Upon Tyne Hospitals NHS Foundation Trust, Newcastle Upon Tyne), Micheal Smith (Royal Devon \& Exeter NHS Foundation Trust, Exeter, Devon), Areeb Moosavi (Milton Keynes University Hospital NHS Foundation Trust, Milton Keynes), Anthony J W King (Nottingham University Hospitals NHS Trust, Nottingham), Matthew Kinsella (Buckinghamshire Healthcare NHS Trust, Stoke Mandeville Hospital, Buckinghamshire).

Disclosures. Francesco Oddone has received consultancy fees from Santen, Allergan, Sooft, Omikron Italia, Centervue and Novartis. Dr Lucia Tanga has no financial disclosures. Dr Péter Kóthy has received lecturing fees from Santen and Novartis. Gábor Holló has received consultancy and lecturing fees from Santen and Novartis.

Compliance with Ethics Guidelines. The study was conducted in accordance with the Declaration of Helsinki of 1964 as revised in 2013. The study protocol was approved by the institutional review board (IRB) or independent ethics committee (IEC) at each center/institution and written informed consent was obtained from subjects prior to enrollment.

Data Availability. The datasets used during and/or analyzed during the current study are available from the corresponding author on reasonable request.

Open Access. This article is licensed under a Creative Commons Attribution-NonCommercial 4.0 International License, which permits any non-commercial use, sharing, adaptation, distribution and reproduction in any medium or format, as long as you give appropriate credit to the original author(s) and the source, provide a link to the Creative Commons licence, and indicate if changes were made. The images or other third party material in this article are included in the article's Creative Commons licence, unless indicated otherwise in a credit line to the material. If material is not included in the article's Creative Commons licence and your intended use is not permitted by statutory regulation or exceeds the permitted use, you will need to obtain permission directly from the copyright holder. To view a copy of this licence, visit http://creativecommons.org/licenses/by$\mathrm{nc} / 4.0 /$.

\section{REFERENCES}

1. International Council of Ophthalmology. Guidelines for glaucoma care. 2016. http://www.icoph.org/ downloads/ICOGlaucomaGuidelines.pdf. Accessed 7 Nov 2019.

2. Collaborative Normal-Tension Glaucoma Study Group. The effectiveness of intraocular pressure reduction in the treatment of normal-tension glaucoma. Collaborative Normal-Tension Glaucoma Study Group. Am J Ophthalmol. 1998;126: 498-505.

3. The AGIS Investigators. The Advanced Glaucoma Intervention Study (AGIS): 7. The relationship between control of intraocular pressure and visual field deterioration. The AGIS Investigators. Am J Ophthalmol. 2000;130:429-40.

4. Heijl A, Leske MC, Bengtsson B, et al. Reduction of intraocular pressure and glaucoma progression: results from the Early Manifest Glaucoma Trial. Arch Ophthalmol. 2002;120:1268-79.

5. Janz NK, Wren PA, Lichter PR, et al. The Collaborative Initial Glaucoma Treatment Study: interim quality of life findings after initial medical or surgical treatment of glaucoma. Ophthalmology. 2001;108:1954-65.

6. European Glaucoma Society. Terminology and guidelines for glaucoma. 2014. https://www.eugs. org/eng/egs_guidelines_download.asp Accessed Oct 2019.

7. Holló G, Katsanos A, Boboridis KG, Irkec M, Konstas AG. Preservative-free prostaglandin analogs and prostaglandin/timolol fixed combinations in the treatment of glaucoma: efficacy, safety and potential advantages. Drugs. 2018;78(1):39-64.

8. Tabet R, Stewart WC, Feldman R, Konstas AG. A review of additivity to prostaglandin analogs: fixed and unfixed combinations. Surv Ophthalmol. 2008;53(1):S85-92.

9. Holló G, Topouzis F, Fechtner RD. Fixed-combination intraocular pressure lowering therapy for glaucoma and ocular hypertension: advantages in clinical practice. Expert Opin Pharmacother. 2014;15(12):1737-47. 
10. Konstas AG, Katsanos A, Athanasopoulos GP, et al. Preservative-free tafluprost/timolol fixed combination: comparative 24-h efficacy administered morning or evening in open-angle glaucoma patients. Expert Opin Pharmacother. 2018;19: 1981-8.

11. Konstas AGP, Haidich AB, Rossetti L, et al. Prostaglandin-timolol fixed combinations efficacy: myth or reality? Editorial. Eur J Ophthalmol. 2012;22:1-4.

12. Aptel F, Cucherat M, Denis P. Efficacy and tolerability of prostaglandin-timolol fixed combinations: a meta-analysis of randomized clinical trials. Eur J Ophthalmol. 2012;22:5-18.

13. Quaranta L, Biagioli E, Riva I, et al. Prostaglandin analogs and timolol-fixed versus unfixed combinations or monotherapy for open-angle glaucoma: a systematic review and meta-analysis. J Ocul Pharmacol Ther. 2013;29:382-9.

14. Pfeiffer N, Traverso CE, Lorenz K, et al. A 6-month study comparing efficacy, safety, and tolerability of the preservative-free fixed combination of tafluprost $0.0015 \%$ and timolol $0.5 \%$ versus each of its individual preservative-free components. Adv Ther. 2014;31:1228-46.

15. Holló G, Hommer A, Antón López A, et al. Efficacy, safety, and tolerability of preservative-free fixed combination of tafluprost $0.0015 \%$ /timolol $0.5 \%$ versus concomitant use of the ingredients. J Ocul Pharmacol Ther. 2014;30:468-75.

16. Holló G, Katsanos A. Safety and tolerability of the tafluprost/timolol fixed combination for the treatment of glaucoma. Expert Opin Drug Saf. 2015;14: 609-17.

17. Kaarniranta K, Ikaheimo K, Mannermaa E, et al. Pharmacokinetics, efficacy, and safety of the preservative-free fixed combination of tafluprost 0 . 0015\% and timolol $0.5 \%$ in healthy volunteers: a phase I comparison vs. the corresponding preservative-free monotherapies. Clin Pharmacokinet. 2016;55:485-94.

18. Hoy SM. Tafluprost/timolol: a review in open-angle glaucoma or ocular hypertension. Drugs. 2015;75: 1807-13.

19. Jaenen N, Baudouin C, Pouliquen P, Manni G, Figueiredo A, Zeyen T. Ocular symptoms and signs with preserved and preservative-free glaucoma medications. Eur J Ophthalmol. 2007;17(3):341-9.

20. Baudouin C. Detrimental effect of preservatives in eyedrops: implications for the treatment of glaucoma. Acta Ophthalmol. 2008;86(7):716-26.
21. Pisella PJ, Pouliquen P, Baudouin C. Prevalence of ocular symptoms and signs with preserved and preservative free glaucoma medication. $\mathrm{Br} \mathrm{J}$ Ophthalmol. 2002;86(4):418-23.

22. Asiedu K, Abu SL. The impact of topical intraocular pressure lowering medications on the ocular surface of glaucoma patients: a review. J Curr Ophthalmol. 2018;31(1):8-15.

23. Aguayo Bonniard A, Yeung JY, Chan CC, Birt CM. Ocular surface toxicity from glaucoma topical medications and associated preservatives such as benzalkonium chloride (BAK). Expert Opin Drug Metab Toxicol. 2016;12(11):1279-89.

24. Boimer C, Birt CM. Preservative exposure and surgical outcomes in glaucoma patients: the PESO study. J Glaucoma. 2013;22:730-5.

25. Leung EW, Medeiros FA, Weinreb RN. Prevalence of ocular surface disease in glaucoma patients. J Glaucoma. 2008;17:350-5.

26. Erb C, Gast U, Schremmer D. German register for glaucoma patients with dry eye. I. Basic outcome with respect to dry eye. Graefes Arch Clin Exp Ophthalmol. 2008;246:1593-601.

27. Garcia-Feijoo J, Sampaolesi JR. A multicentre evaluation of ocular surface disease prevalence in patients with glaucoma. Clin Ophthalmol. 2012;6: 441-6.

28. Spitzer E, Cannon CP, Serruys PW. Should realworld evidence be incorporated into regulatory approvals? Expert Opin Drug Saf. 2018;17:1155-9.

29. Eichler HG, Bloechl-Daum B, Broich K, et al. Data rich, information poor: can we use electronic health records to create a learning healthcare system for pharmaceuticals? Clin Pharmacol Ther. 2019;105(4):912-22.

30. Food and Drug Administration. Use of real-world evidence to support regulatory decision-making for medical devices: guidance for industry and Food and Drug Administration staff. 2018. http://www. fda.gov/downloads/MedicalDevices/ DeviceRegulationandGuidance/ GuidanceDocuments/UCM513027.pdf. Accessed 7 Nov 2019.

31. NIDEK. Conversion table for representation of visual acuity. https://www.nidek-intl.com/visual_ acuity.html. Accessed 7 Nov 2019.

32. Bron AJ, Evans VE, Smith JA. Grading of corneal and conjunctival staining in the context of other dry eye tests. Cornea. 2003;22(7):640-50. 
33. Bhapkar V. A note on the equivalence of two test criteria for hypotheses in categorical data. J Am Stat Assoc. 1966;61(313):228-35.

34. Holló G, Vuorinen J, Tuominen J, Huttunen T, Ropo A, Pfeiffer N. Fixed-dose combination of tafluprost and timolol in the treatment of openangle glaucoma and ocular hypertension: comparison with other fixed-combination products. Adv Ther. 2014;31(9):932-44. 